\title{
Método dos Elementos de Contorno Aplicado a Problemas de Laplace: Comparação em Problemas Setorialmente Homogêneos entre a Técnica das Sub-regiões e uma Nova Técnica com Sobreposição de Domínios
}

\author{
Andrade, A. J. C $^{1 *}$; Loeffler, C. F. ${ }^{1}$ \\ 1 Programa de Pós-Graduação em Engenharia Mecânica, Universidade Federal do Espírito Santo, Vitória, ES, Brasil. \\ *e-mail: andrejudah@gmail.com
}

\begin{abstract}
Resumo
O Método dos Elementos de Contorno (MEC) tem excelente desempenho nas aplicações em que o campo de variáveis é escalar e estacionário. No entanto, há uma gama de problemas nas ciências exatas e na engenharia que são sabidamente difíceis de serem resolvidos pelo MEC. Entre estes, estão os problemas fisicamente não homogêneos, onde as propriedades físicas podem variar localmente ou setorialmente. Para esses problemas, as formulações de domínio, como os Métodos de Elementos Finitos (MEF), Volumes Finitos (MVF) ou mesmo Diferenças Finitas (MDF), apresentam vantagens consideráveis. Entretanto, mesmo para estes casos, existe um procedimento clássico e consistente através do MEC, que é a técnica das sub-regiões. Neste trabalho, apresenta-se a comparação entre esta técnica e uma nova alternativa que se baseia na sobreposição de domínios, tendo como referência resultados analíticos e resultados obtidos através do Método dos Elementos Finitos.
\end{abstract}

Palavras chaves: Método dos Elementos de Contorno. Homogeneidade Setorial. Equação de Laplace

\begin{abstract}
The Boundary Element Method (BEM) has excellent performance in applications where the variable field is scalar and stationary. However, there is a wide range of issues in science and engineering that are difficult to solve by the BEM. Among these issues, there are the non-homogeneous media problems, where the physical properties vary locally. In these kind of problems, the domain techniques, such as Finite Element Method (FEM), Finite Volume Method (FVM) or Finite Difference Method (FDM), present considerable advantages. However, even for these cases, it is possible to obtain consistent formulations for BEM, as the technique of subregions. This paper presents a comparison between this technique and a new alternative that is based on domain overlapping. The reference used in this work are analytical results and results obtained by the Finite Element Method.
\end{abstract}

Keywords: Boundary Element Method. Non-homogeneous Media. Laplace Equation.

\section{Introdução}

A Equação de Laplace é a equação mais simples na Teoria de Campo Escalar. Nos problemas representados por essa equação, as ações externas atuam apenas pelo contorno. Assim, essas ações, que não são apresentadas explicitamente na equação, são expressas como condições de contorno do problema. Tal equação é dada por:

$$
\mathrm{Ku}_{, \mathrm{ii}}=0
$$

Na Equação (1), K representa as propriedades do domínio em estudo. Ao se considerar o domínio com propriedades isotrópicas, diz-se que o problema é fisicamente homogêneo e esta constante poderia ser omitida. Porém, caso haja consideração de que as propriedades materiais de um domínio variem, a modelagem do problema deve ser realizada considerando-se conhecida a forma de variação ao longo de todo o domínio. 
Chamam-se de problemas fisicamente nãohomogêneos aqueles em que a propriedade $K$ varia apenas espacialmente. Nos casos onde a propriedade $\mathrm{K}$ tem valores constantes, porém em diferentes setores macroscópicos do domínio, diz-se que o problema apresenta homogeneidade setorial. Nesta classe de problemas, os métodos numéricos de domínio se adaptam facilmente, pois em cada subdivisão do domínio pode-se modelar a propriedade $\mathrm{K}$ com um valor constante. Porém, existe relativa dificuldade em modelar essa classe de problemas através de métodos que utilizam a discretização do contorno.

A técnica das sub-regiões é uma alternativa já utilizada no âmbito do Método dos Elementos de Contorno, onde as regiões de propriedades distintas têm suas matrizes montadas de forma independente e o sistema de equações para todo o domínio é montado a partir de um subconjunto de equações gerado em cada subregião, utilizando condições de compatibilidade e equilíbrio em suas interfaces [1]. Outras possibilidades, utilizando o Método dos Elementos de Contorno, seria a utilização da formulação Quase-Dupla Reciprocidade [2], onde a formulação do MEC é trabalhada no seu equacionamento, introduzindo uma aproximação nos termos integrais de derivada espacial de primeira ordem. Esta formulação já foi aplicada a problemas dinâmicos em meios heterogêneos, mas em situações nas quais as propriedades físicas variam gradativamente [3].

\section{Equação Integral do MEC}

A formulação integral do MEC se baseia na utilização de ferramentas matemáticas básicas da teoria das equações integrais, com base na equação integral associada à Equação (1), utilizando como função auxiliar a solução fundamental [1], e integrando sobre o domínio físico $\Omega(\mathrm{X})$, como mostrado a seguir:

$$
\int_{\Omega} \mathrm{Ku}_{, \mathrm{ii}}(\mathrm{X}) \mathrm{u}^{*}(\xi ; \mathrm{X}) \mathrm{d} \Omega=0
$$

Com a aplicação de integração por partes e o teorema da divergência na Equação (2), operações bem documentadas na literatura $[1,4]$, chega-se a seguinte expressão integral:

$$
\begin{aligned}
& \mathrm{K}\left[\int_{\Gamma} \mathrm{q}(\mathrm{X}) \mathrm{u}^{*}(\xi ; \mathrm{X}) \mathrm{d} \Gamma-\int_{\Gamma} \mathrm{u}(\mathrm{X}) \mathrm{q}^{*}(\xi ; \mathrm{X}) \mathrm{d} \Gamma+\right. \\
& \left.\int_{\Omega} \mathrm{u}(\mathrm{X}) \mathrm{u}_{, \mathrm{ii}}^{*}(\xi ; \mathrm{X}) \mathrm{d} \Omega\right]=0
\end{aligned}
$$

Considerando as funções auxiliares $u^{*}(\xi ; X)$ e sua derivada normal derivado $q^{*}(\xi ; X)$, onde $\xi$ é um ponto do domínio denominado ponto fonte, que são obtidas a partir da solução do problema fundamental, dado por:

$$
\mathrm{u}_{, \mathrm{ii}}^{*}(\xi ; \mathrm{X})=-\Delta(\xi ; \mathrm{X})
$$

Onde $\Delta$ é a função delta de Dirac. A substituição da Equação (3) na Equação (4), e utilizando as propriedades da função delta de Dirac, fornece a seguinte equação integral de contorno:

$$
\begin{aligned}
& K\left[\int_{\Gamma} q(X) u^{*}(\xi ; X) d \Gamma-\int_{\Gamma} u(X) q^{*}(\xi ; X) d \Gamma+\right. \\
& c(\xi) u(\xi)]=0
\end{aligned}
$$

Na Equação (5), o valor do coeficiente $c(\xi)$ depende não só sobre o posicionamento do ponto fonte $\xi$ com respeito ao domínio físico $\Omega(\mathrm{X})$, mas também sobre a suavidade do contorno neste ponto $[1,4]$.

A partir da Equação (5) pode-se proceder ao processo de discretização do contorno, escolhendo adequadamente pontos onde posicionar $\xi$ de forma a se obter um sistema de equações que resolva as incógnitas no contorno - os pontos nodais. Ao mesmo tempo, é preciso também arbitrar uma aproximação para o campo de variáveis ( $\mathrm{u}$ e q) entre os nós gerados pela discretização.

O sistema linear gerado pela discretização do contorno é um sistema que têm incógnitas tanto na variável básica quanto na sua derivada, tendo a seguinte representação matricial:

$$
\mathrm{K}[\mathrm{H}]\{\mathrm{U}\}=\mathrm{K}[\mathrm{G}]\{\mathrm{Q}\}
$$

Sendo $[H]$ e $[G]$ matrizes $n \times n$ e $\{U\}$ e $\{Q\}$ vetores de tamanho $\mathrm{n}$, sendo $\mathrm{n}$ o número de incógnitas da discretização do contorno.

\section{Técnica das Sub-Regiões}

O método das sub-regiões é utilizado dentro do MEC como alternativa para resolução de problemas onde há homogeneidade setorial. A Figura 1 ilustra um domínio $\Omega$ com duas sub-regiões com propriedades distintas, $\mathrm{K}_{1}$ em $\Omega_{1}$ e K $\mathrm{K}_{2}$ em $\Omega_{2}$.

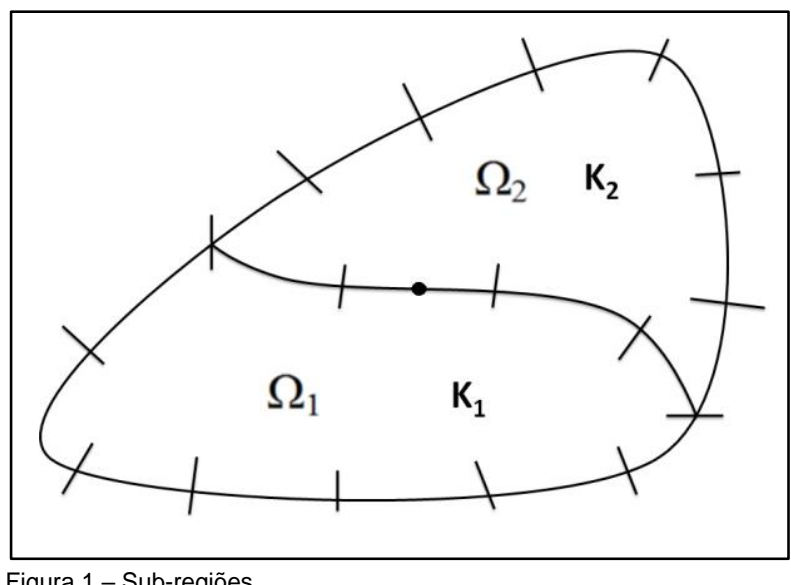


O sistema de equações resultante é formado pela adição dos sistemas isolados referentes a cada subregião. As equações que permitem este acoplamento em conjunto são as condições de compatibilidade e equilíbrio na interface entre as sub-regiões [1,4]. Por exemplo, em cada ponto comum na interface de duas sub-regiões, conforme Figura 2, tem-se:

Condição de compatibilidade: $\mathrm{u}_{12}=\mathrm{u}_{21}$.

Condição de equilíbrio: $Q_{12}=K_{1} q_{12}=-K_{2} q_{21}=-Q_{21}$.

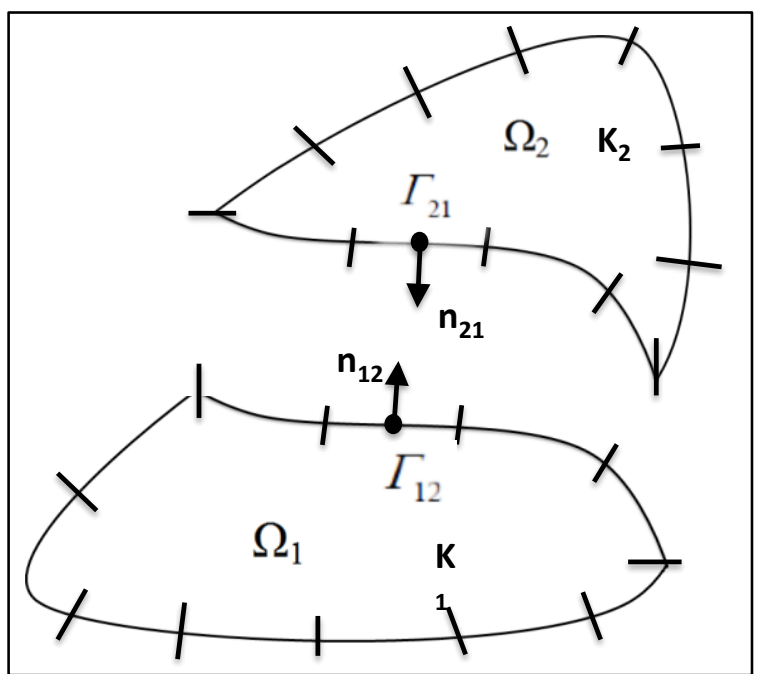

Figura 2 - Condições de acoplamento.

O sistema de equações correspondente às sub-regiões pode ser escrito como:

$$
\begin{aligned}
& {\left[\begin{array}{ll}
\mathrm{H}^{1} & \mathrm{H}_{\mathrm{I}}^{1}
\end{array}\right]\left\{\begin{array}{l}
\mathrm{U}^{1} \\
\mathrm{U}_{\mathrm{I}}^{1}
\end{array}\right\}=\left[\begin{array}{ll}
\mathrm{G}^{1} & \mathrm{G}_{\mathrm{I}}^{1}
\end{array}\right]\left\{\begin{array}{l}
\mathrm{Q}^{1} \\
\mathrm{Q}_{\mathrm{I}}^{1}
\end{array}\right\}} \\
& {\left[\begin{array}{ll}
\mathrm{H}^{2} & \mathrm{H}_{\mathrm{I}}^{2}
\end{array}\right]\left\{\begin{array}{l}
\mathrm{U}^{2} \\
\mathrm{U}_{\mathrm{I}}^{2}
\end{array}\right\}=\left[\begin{array}{ll}
\mathrm{G}^{2} & \mathrm{G}_{\mathrm{I}}^{2}
\end{array}\right]\left\{\begin{array}{l}
\mathrm{Q}^{2} \\
\mathrm{Q}_{\mathrm{I}}^{2}
\end{array}\right\}}
\end{aligned}
$$

Aplicando as condições de compatibilidade e equilíbrio na interface e combinando os sistemas de equações:

$$
\left[\begin{array}{ccc}
\mathrm{H}^{1} & \mathrm{H}_{\mathrm{I}}^{1} & 0 \\
0 & \mathrm{H}_{\mathrm{I}}^{2} & \mathrm{H}^{2}
\end{array}\right]\left\{\begin{array}{c}
\mathrm{U}^{1} \\
\mathrm{U}_{\mathrm{I}} \\
\mathrm{U}^{2}
\end{array}\right\}=\left[\begin{array}{ccc}
\mathrm{G}^{1} & \mathrm{G}_{\mathrm{I}}^{1} & 0 \\
0 & -\mathrm{G}_{\mathrm{I}}^{2} & \mathrm{G}^{2}
\end{array}\right]\left\{\begin{array}{l}
\mathrm{Q}^{1} \\
\mathrm{Q}_{\mathrm{I}} \\
\mathrm{Q}^{2}
\end{array}\right\}
$$

Uma característica da matriz resultante deste sistema é ser uma matriz em banda e que quanto mais interfaces existem no domínio, mais zeros haverá na matriz.

\section{Técnica da Partição}

A abordagem via sub-regiões impõe cada subdomínio sendo examinado de modo estanque. Seu acoplamento ao domínio como um todo é feito através das condições de continuidade e equilíbrio, conforme apresentado anteriormente.
Na presente formulação, diferentemente do que se faz nas sub-regiões, o domínio envolvente $\Omega$ não precisa ser subtraído de qualquer parte, conforme ilustra a Figura 3, representando matematicamente através da Equação (10).

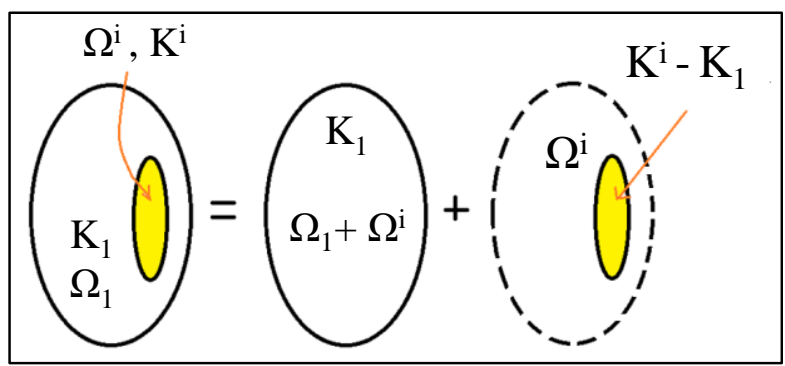

Figura 3 - Procedimento proposto.

$$
\int_{\Omega} \mathrm{Kd} \Omega=\int_{\Omega} \mathrm{K}_{1} \mathrm{~d} \Omega+\int_{\Omega \mathrm{i}} \mathrm{K}^{*} \mathrm{~d} \Omega=0
$$

Sendo $K^{*}=K^{i}-K_{1}$.

Nesta abordagem, cada domínio se mantém com propriedades homogêneas.

A partir da Equação de Laplace, desenvolve-se a formulação proposta:

$$
\int_{\Omega} \mathrm{K}_{1} \mathrm{u}_{, \mathrm{ii}} \mathrm{u}^{*} \mathrm{~d} \Omega+\int_{\Omega \mathrm{i}} \mathrm{K}^{*} \mathrm{u}_{, \mathrm{ii}}^{\mathrm{i}} \mathrm{u}^{*} \mathrm{~d} \Omega=0
$$

O subscrito i enfatiza que se trata de valores do potencial referentes ao contorno interno. Ressalta-se então, que nesta formulação, pontos fonte internos devem figurar explicitamente no sistema matricial do MEC. Usualmente, tais pontos fonte são tratados como dados de entrada para o reuso da equação integral e cálculo das variáveis de estado no interior do domínio, isto após a solução do sistema matricial dado pela Equação (6).

A primeira parcela do lado esquerdo da Equação (11) pode ser desenvolvida conforme procedimentos normais do MEC, assim:

$$
\begin{gathered}
\int_{\Omega} \mathrm{K}_{1} \mathrm{u}_{, \mathrm{ii}} \mathrm{u}^{*} \mathrm{~d} \Omega=\int_{\Gamma} \mathrm{K}_{1} \mathrm{qu}^{*} \mathrm{~d} \Gamma-\int_{\Gamma} \mathrm{K}_{1} \mathrm{uq} \mathrm{q}^{*} \mathrm{~d} \Gamma- \\
\mathrm{u}(\xi) \mathrm{c}(\xi) \mathrm{K}_{1}(\xi)
\end{gathered}
$$

Desenvolvendo a segunda parcela da Equação (11) de forma similar:

$$
\begin{array}{r}
\int_{\Omega i} K^{*} u_{, i i}^{i} u^{*} d \Omega=\int_{\Gamma i} K^{*} q^{i} u^{*} d \Gamma- \\
\int_{\Gamma i} K^{*} u^{i} q^{*} d \Gamma-u^{i}(\xi) c(\xi) K^{*}(\xi)
\end{array}
$$

No desenvolvimento da formulação proposta, o valor de $c(\xi)$ na Equação (13) é dependente da forma como 
setor interno $\left(\Omega^{\mathrm{i}}\right)$ se localiza em relação ao domínio $(\Omega)$, ou seja, se há ou não pontos de interseção entre os contornos. No caso do contorno interno $\Gamma^{\mathrm{i}}$ estar completamente envolvido pelo primeiro, os pontos fontes $\xi$ são posicionados exclusivamente no contorno externo $\Gamma^{\mathrm{i}}$ e o valor de $\mathrm{c}(\xi)$ é nulo. É o caso mostrado na Figura 4. A caracterização do contorno $\Gamma^{\mathrm{i}}$ é feita pelas coordenadas destes pontos.

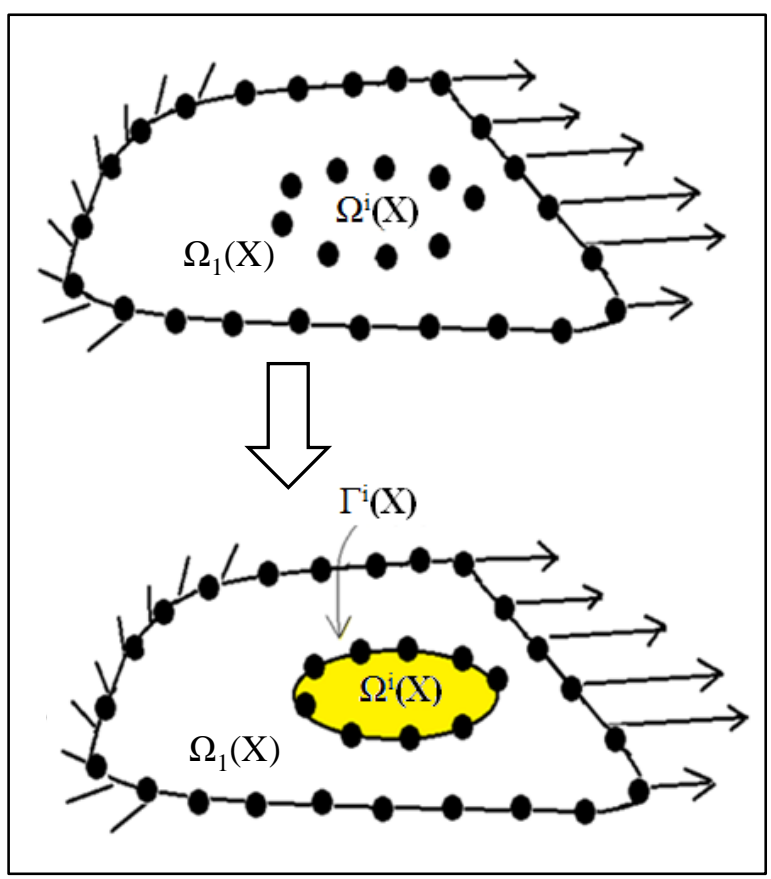

Figura 4 - llustração de um setor restrito $\Omega$ i totalmente imerso no domínio $\Omega$.

Para o caso de haver interseção, o valor de $c(\xi)$ vai depender da suavidade do contorno $[1,4]$.

Por conveniência, considere o caso dado na Figura 4, onde o valor de $c(\xi)$ na Equação (13) é zero, pois $\xi$ esta localizado fora do contorno $\Gamma^{i}$. Não há perda de generalidade procedendo desta forma. Assim, a partir das Equações (12) e (13):

$$
\begin{gathered}
\int_{\Gamma} \mathrm{qu}^{*} \mathrm{~d} \Gamma-\int_{\Gamma} \mathrm{uq^{* }} d \Gamma-\mathrm{u}(\xi) \mathrm{c}(\xi)=-\frac{\mathrm{K}^{*}}{\mathrm{~K}_{1}}\left(\int_{\Gamma \mathrm{i}} \mathrm{q}^{\mathrm{i}} \mathrm{u}^{*} \mathrm{~d} \Gamma-\right. \\
\left.\int_{\Gamma \mathrm{i}} \mathrm{u}^{\mathrm{i}} \mathrm{q}^{*} \mathrm{~d} \Gamma\right)
\end{gathered}
$$

Pode-se interpretar o lado direito da Equação (14) através dos princípios de energia, pois as suas duas integrais representam, respectivamente, contribuições de energia vindas dos fluxos atuantes e da energia difusiva do setor interno. Tais contribuições afetam o balanço de energia total, em que também as parcelas difusiva e de fluxo relacionadas ao contorno envolvente $\Gamma$ precisam ser contabilizadas. Poder-se-ia expressar a influência do setor heterogêneo no domínio completo através da energia difusiva ou dos fluxos de calor; contudo é muito mais simples transportar a influência do setor interno através da energia difusiva, que vem expressa em termos dos potenciais $u^{i}$, do que pelos fluxos $q^{i}$.

Assim, por conveniência, neste modelo proposto, a energia armazenada proveniente do setor heterogêneo é dada apenas pelos potenciais internos $u^{i}$. Desse modo, apenas uma parcela do lado direito da Equação (14) é computada:

$$
\int_{\Gamma} \mathrm{qu}^{*} \mathrm{~d} \Gamma-\int_{\Gamma} \mathrm{uq}^{*} \mathrm{~d} \Gamma-\mathrm{u}(\xi) \mathrm{c}(\xi)=\frac{\mathrm{K}^{*}}{\mathrm{~K}_{1}}\left(\int_{\Gamma \mathrm{i}} \mathrm{u}^{\mathrm{i}} \mathrm{q}^{*} \mathrm{~d} \Gamma\right)
$$

Em forma matricial, podemos representar da seguinte forma:

$$
[\mathrm{H}]\{\mathrm{U}\}=[\mathrm{G}]\{\mathrm{Q}\}+\left[\mathrm{H}^{\mathrm{i}}\right]\left\{\mathrm{U}^{\mathrm{i}}\right\}
$$

Os pontos internos que envolvem o contorno interno $\Gamma^{\mathrm{i}}$ devem ser representados no sistema matricial do MEC, representando valores incógnitos de potencial, tal como ocorre na MECDR e na MECID para melhorar a qualidade da interpolação das ações de domínio [5].

Sendo a energia proveniente do setor heterogêneo representada pela energia difusiva, o acoplamento do sistema completo de equações é facilitada, pois é realizada através dos valores dos potenciais nos pontos internos, ao invés de seus fluxos. Gera-se uma matriz setorial $\mathrm{H}^{\mathrm{i}}$ que pode ser facilmente contabilizada no sistema final. Nela aparecem explicitamente os pontos internos incógnitas no sistema final de equações, ou seja:

$$
\left[\begin{array}{cc}
\mathrm{H}_{\mathrm{cc}} & 0_{\mathrm{ci}} \\
\mathrm{H}_{\mathrm{ic}} & \mathrm{H}_{\mathrm{ii}}
\end{array}\right]\left\{\begin{array}{l}
\mathrm{u}_{\mathrm{c}} \\
\mathrm{u}_{\mathrm{i}}
\end{array}\right\}=\left[\begin{array}{cc}
\mathrm{G}_{\mathrm{cc}} & 0_{\mathrm{ci}} \\
\mathrm{G}_{\mathrm{ic}} & 0_{\mathrm{ii}}
\end{array}\right]\left\{\begin{array}{l}
\mathrm{q}_{\mathrm{c}} \\
\mathrm{q}_{\mathrm{i}}
\end{array}\right\}+\left[\mathrm{H}^{\mathrm{i}}\right]\left\{\mathrm{U}^{\mathrm{i}}\right\}
$$

A parcela do lado direito da Equação (15) é referente ao domínio $\Omega^{\mathrm{i}}$, e são conectadas ao domínio completo $\Omega$ através da integração realizada no contorno $\Gamma^{\mathrm{i}}$ com os pontos fonte localizados no contorno externo $\Gamma$. Assim leva-se a informação do contorno interno $\Gamma^{\mathrm{i}}$ ao externo Г. Representando matricialmente:

$$
\left[\begin{array}{ll}
\mathrm{H}_{\mathrm{cc}} & \mathrm{H}_{\mathrm{ci}} \\
\mathrm{H}_{\mathrm{ic}} & \mathrm{H}_{\mathrm{ii}}
\end{array}\right]\left\{\begin{array}{l}
\mathrm{u}_{\mathrm{c}} \\
\mathrm{u}_{\mathrm{i}}
\end{array}\right\}=\left[\begin{array}{cc}
\mathrm{G}_{\mathrm{cc}} & 0_{\mathrm{ci}} \\
\mathrm{G}_{\mathrm{ic}} & 0_{\mathrm{ii}}
\end{array}\right]\left\{\begin{array}{l}
\mathrm{q}_{\mathrm{c}} \\
\mathrm{q}_{\mathrm{i}}
\end{array}\right\}
$$

A matriz $\mathrm{H}_{\mathrm{ci}}$ representa os coeficientes gerados pela integração do contorno interno $\Gamma^{i}$ com os pontos fontes localizados no contorno externo $\Gamma$. Assim é realizada a transmissão da informação do setor $\Omega^{i}$ para o domínio completo $\Omega$.

\section{Simulação Numérica}

Para possibilitar a comparação entre a nova formulação apresentada e o método das sub-regiões no MEC, foram realizadas simulações, utilizando exemplos com 
geometria regular e carregamento simples, para comparação dos resultados em termos de erros relativos.

Os valores de erros foram calculados utilizando resultados de referência a partir da a formula de erro da Equação (19).

$$
\text { Erro }=\sum_{n=1}^{N} \frac{\left|u_{\text {referência }}-u_{\text {simuladoo }}\right|}{N * \text { valor max }\left(u_{\text {referência }}\right)}
$$

\subsection{Exemplo 1}

A primeira simulação apresenta um domínio com dois setores com propriedades distintas. A geometria do problema, assim como as condições de contorno e os valores das propriedades de cada setor são mostrados na Figura 5.

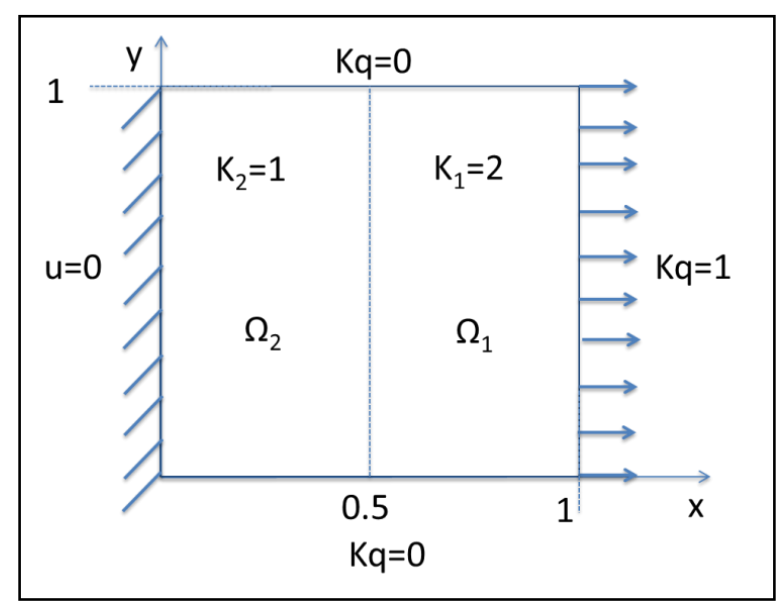

Figura 5: Domínio com setores $\Omega_{1}$ e $\Omega_{2}$.

A geometria foi simulada no programa de elementos de contorno com os seguintes números de elementos: 8 , $16,32,64$ e 128. O erro relativo entre os resultados analíticos e os valores simulados pelas técnicas de sub-região e nova formulação no cálculo do potencial é apresentado na Figura 6 e na Tabela 1.

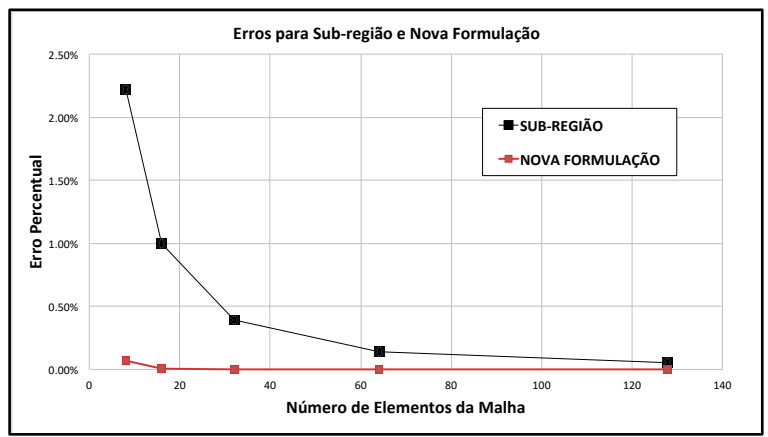

Figura 6: Erro relativo $X$ Numero de Elementos da malha para SubRegião e Nova Formulação.

\begin{tabular}{|c|c|c|}
\hline $\begin{array}{l}\text { Número de } \\
\text { Elementos }\end{array}$ & $\begin{array}{c}\text { SUB- } \\
\text { REGIÃO }\end{array}$ & $\begin{array}{c}\text { NOVA } \\
\text { FORMULAÇÃO }\end{array}$ \\
\hline 8 & $2.219 \%$ & $0.068 \%$ \\
\hline 16 & $1.000 \%$ & $0.003 \%$ \\
\hline 32 & $0.395 \%$ & $0.001 \%$ \\
\hline 64 & $0.143 \%$ & $0.0004 \%$ \\
\hline 128 & $0.050 \%$ & $0.0001 \%$ \\
\hline
\end{tabular}

A partir desses resultados é possível evidenciar que, para ambas as técnicas, o valor do erro diminui com o aumento do numero de elementos da malha, porém, o ponto de maior relevância é a curva de erros observada para a nova formulação. Mesmo com uma discretização grosseira, os valores de erro relativos são muito reduzidos. Essa tendência se mantém com o aumento do numero de elementos.

\subsection{Exemplo 2}

Utilizou-se uma configuração geométrica com duas regiões com propriedades distintas, uma imersa na outra. A Figura 7 mostra a geometria simulada com as condições de contorno utilizadas.

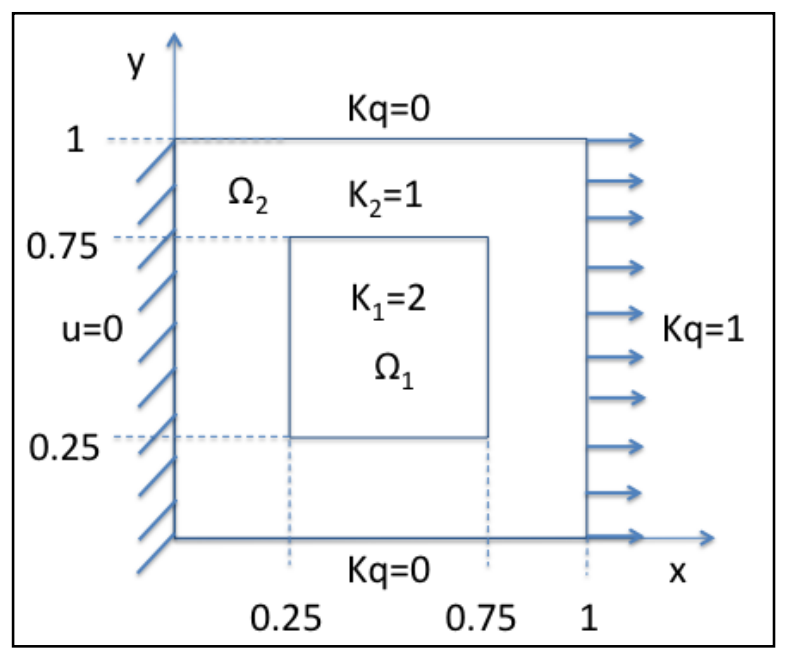

Figura 7: Domínio $\Omega_{2}$ com setor $\Omega_{1}$.

Este exemplo é bem representativo para a comparação entre as técnicas do MEC visto o setor heterogêneo $\left(\Omega_{1}\right)$ estar totalmente imerso no domínio $\Omega_{2}$. Devido à geometria bidimensional e as diferentes propriedades físicas dos setores, este problema não tem solução analítica simples. A solução de referência utilizada foi obtida por simulações realizadas através do Método dos Elementos Finitos (MEF) utilizando uma malha estruturada com 1.681 nós e 3.200 elementos.

Para as simulações com a técnica das sub-regiões foram realizadas simulações com 12, 32, 64 e 128 elementos de contorno. Os resultados foram comparados com as simulações realizadas através do Método dos Elementos Finitos (MEF), ao longo de uma linha do domínio em $y=1$. $O$ erro relativo entre os 
resultados obtidos através do MEF e os valores simulados pelas técnicas de sub-região e nova formulação são apresentados na Figura 8.

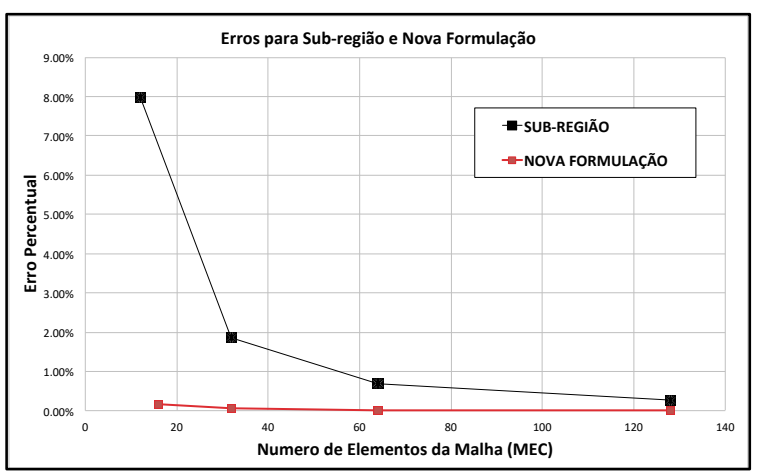

Figura 8: Erro relativo $\mathrm{X}$ Numero de Elementos da malha para SubRegião e Nova Formulação.

Tabela 2: Erro Percentual - Simulação exemplo 2.

\begin{tabular}{c|c|c}
\hline $\begin{array}{c}\text { Número de } \\
\text { Elementos }\end{array}$ & $\begin{array}{c}\text { SUB- } \\
\text { REGIÃO }\end{array}$ & $\begin{array}{c}\text { NOVA } \\
\text { FORMULAÇÃO }\end{array}$ \\
\hline 12 & $7.981 \%$ & $0.175 \%$ \\
\hline 32 & $1.865 \%$ & $0.052 \%$ \\
\hline 64 & $0.695 \%$ & $0.011 \%$ \\
\hline 128 & $0.259 \%$ & $0.005 \%$ \\
\hline
\end{tabular}

O resultado apresentado é similar ao encontrado no exemplo anterior. Para ambas as técnicas, o valor do erro diminui com o aumento do numero de elementos da malha, como esperado devido ao aumento da quantidade de pontos nodais. Porém, novamente, o ponto de maior relevância é a curva de erros observado para a nova formulação. Mesmo com uma baixa discretização, os valores de erro relativos são muito baixos. Essa tendência se mantém com o aumento do numero de elementos. Ressalta-se que este exemplo, por conta da sua bidimensionalidade, é numericamente mais complexo do que o anterior.

\section{Conclusões}

Os resultados revelam-se bastante favoráveis à nova técnica e indicam que ela pode ser facilmente utilizada e replicada nos problemas em que as propriedades físicas do domínio variam setorialmente. Os resultados em termos de erros relativos foram superiores para a nova técnica.

Além da boa precisão, a nova técnica mostra uma enorme vantagem em termos de implementação computacional e de entrada de dados devido a sua simplicidade. Para implementar o modelo, é necessário apenas criar uma nova matriz $\mathrm{H}^{\mathrm{i}}$, referida a energia contida nos setores internos.
A presente técnica não introduz aproximação adicional no modelo numérico, que no caso da técnica clássica de sub-regiões está relacionada com a interpolação adicional sobre os elementos de interfaces do contorno interno.

O procedimento também não cria qualquer impedimento para a implementação de procedimentos auxiliares como a Técnica da Dupla Reciprocidade, o DIBEM ou qualquer outro programa adequado para abordar estas classes mais avançadas de problemas.

Existe nesta nova técnica um potencial significativo no que tange a futuras aplicações, de grande interesse e complexidade, particularmente na análise elastoplástica e na dinâmica estrutural.

Este trabalho teve como escopo o estudo da Equação de Laplace em duas dimensões, trabalhos futuros simples podem ser realizados tratando de equações escalares correlatas, como a Equação de Poisson e a Equação de Helmholtz, que pertencem a Equação de Campo Generalizada.

\section{Referências}

[1] BREBBIA, C. A; WALKER, S. Boundary element techniques in engineering. Elsevier, 1980.

[2] LOEFFLER, C. F.; MANSUR, W. J. Quasi-Dual Reciprocity Boundary Element Formulation for Incompressible Flow: Application to the DiffusiveAdvective Equation. International Journal for Numerical Methods in Engineering. V. 58, Issue 8, p. 1167-1186, John Wiley and Sons, 2003.

[3] SANTOLIN, W. D. Análise dinâmica de problemas escalares não homogêneos através do método dos elementos de contorno. Dissertação (Mestrado em Engenharia Mecânica) - Programa de Pós-Graduação em Engenharia Mecânica, Universidade federal do Espírito Santo, Vitória, 2006.

[4] BREBBIA, C. A.; DOMINGUEZ, J. Boundary elements: an introductory course. WIT press, 1996.

[5] LOEFFLER, C. F.; CRUZ, A. L.; BULCÃO, A. Direct Use of Radial Basis Interpolation Functions for Modelling Source Terms with the Boundary Element Method. Engineering Analysis with Boundary Elements, vol. 50, pp. 97-108, 2015 